\author{
SANDIA REPORT \\ SAND2006-5856 \\ Unlimited Release \\ Printed September 2006
}

\title{
Generating Nonlinear FM Chirp Waveforms for Radar
}

\author{
Armin W. Doerry
}

Prepared by

Sandia National Laboratories

Albuquerque, New Mexico 87185 and Livermore, California 94550

Sandia is a multiprogram laboratory operated by Sandia Corporation, a Lockheed Martin Company, for the United States Department of Energy's

National Nuclear Security Administration under Contract DE-AC04-94AL85000.

Approved for public release; further dissemination unlimited.

\section{Sandia National Laboratories}


Issued by Sandia National Laboratories, operated for the United States Department of Energy by Sandia Corporation.

NOTICE: This report was prepared as an account of work sponsored by an agency of the United States Government. Neither the United States Government, nor any agency thereof, nor any of their employees, nor any of their contractors, subcontractors, or their employees, make any warranty, express or implied, or assume any legal liability or responsibility for the accuracy, completeness, or usefulness of any information, apparatus, product, or process disclosed, or represent that its use would not infringe privately owned rights. Reference herein to any specific commercial product, process, or service by trade name, trademark, manufacturer, or otherwise, does not necessarily constitute or imply its endorsement, recommendation, or favoring by the United States Government, any agency thereof, or any of their contractors or subcontractors. The views and opinions expressed herein do not necessarily state or reflect those of the United States Government, any agency thereof, or any of their contractors.

Printed in the United States of America. This report has been reproduced directly from the best available copy.

Available to DOE and DOE contractors from

U.S. Department of Energy

Office of Scientific and Technical Information

P.O. Box 62

Oak Ridge, TN 37831

Telephone: $\quad$ (865) 576-8401

Facsimile: $\quad$ (865) 576-5728

E-Mail: $\quad$ reports@adonis.osti.gov

Online ordering: http://www.osti.gov/bridge

Available to the public from

U.S. Department of Commerce

National Technical Information Service

5285 Port Royal Rd.

Springfield, VA 22161

Telephone: $\quad$ (800) 553-6847

Facsimile: (703) 605-6900

E-Mail: $\quad$ orders@ntis.fedworld.gov

Online order: $\quad$ http://www.ntis.gov/help/ordermethods.asp?loc=7-4-0\#online

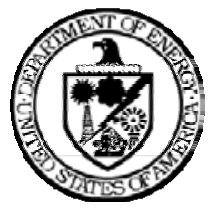


SAND2006-5856

Unlimited Release

Printed September 2006

\title{
Generating Nonlinear FM Chirp Waveforms for Radar
}

\author{
Armin W. Doerry \\ SAR Applications Department \\ Sandia National Laboratories \\ PO Box 5800 \\ Albuquerque, NM 87185-1330
}

\begin{abstract}
Nonlinear FM waveforms offer a radar matched filter output with inherently low range sidelobes. This yields a 1-2 dB advantage in Signal-to-Noise Ratio over the output of a Linear FM waveform with equivalent sidelobe filtering. This report presents design and implementation techniques for Nonlinear FM waveforms.
\end{abstract}




\section{ACKNOWLEDGEMENTS}

A special thanks to Fred Dickey for sharing his insight in fruitful discussions on this topic. Good work is much easier when it can be explored with good people.

This work was funded by the US DOE Office of Nonproliferation \& National Security, Office of Research and Development, NA-22, under the Advanced Radar System (ARS) project.

Sandia is a multiprogram laboratory operated by Sandia Corporation, a Lockheed Martin Company, for the United States Department of Energy under Contract DE-AC0494AL85000. 


\section{CONTENTS}

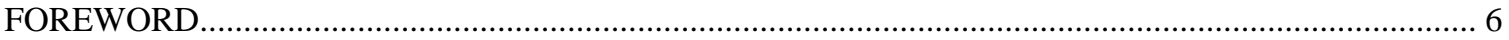

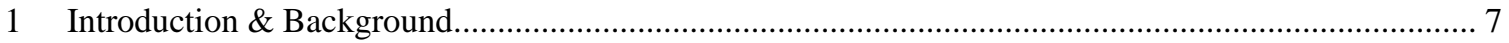

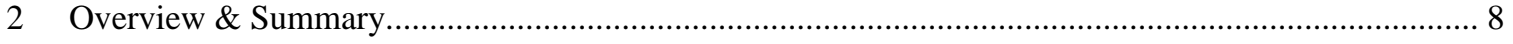

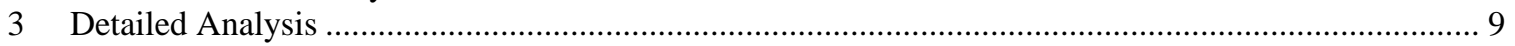

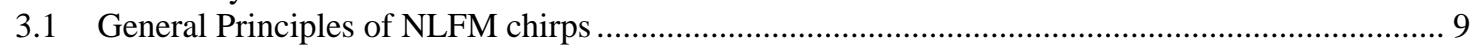

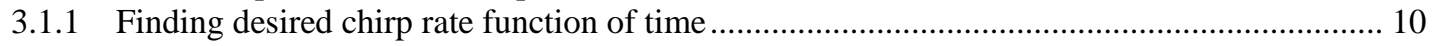

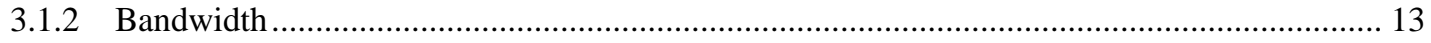

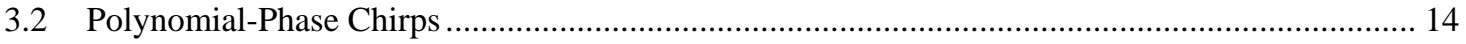

3.2.1 Determining Phase Polynomial Coefficients ........................................................... 16

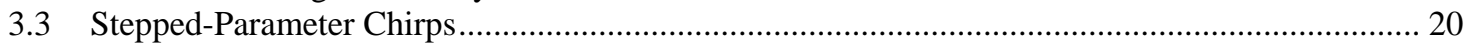

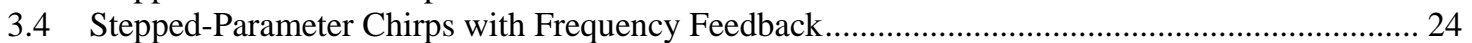

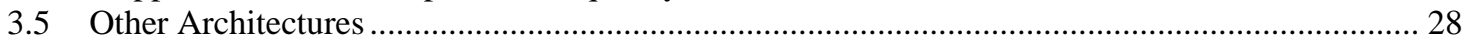

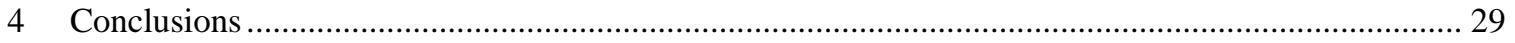

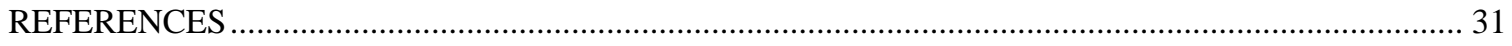

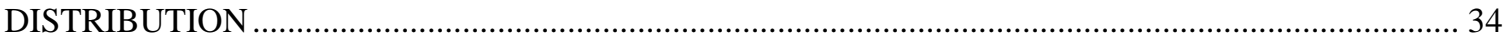




\section{FOREWORD}

Often, especially for power-starved radar systems, the radar designer strives to extract every bit of performance that he is able to coax from his system. A single $\mathrm{dB}$ of additional Signal-to-Noise Ratio (SNR) gained elsewhere is equivalent to a $25 \%$ increase in transmitter power. Alternatively, a single $\mathrm{dB}$ of additional SNR can have dramatic effects in reducing false alarm rates in target detection applications. Consequently we examine herein choosing and creating Nonlinear FM radar waveforms with characteristics that can avoid the typical 1-2 dB of SNR degradation associated with sidelobe filtering that is often required with Linear FM waveforms. 


\section{Introduction \& Background}

It is well known that when a signal is input to a Matched Filter (matched to the input signal) then the output of the filter is the autocorrelation function of the signal. Also well known is that the autocorrelation function is the Fourier Transform of the signal's Power Spectral Density (PSD). A Matched Filter provides optimum (maximum) Signal to Noise Ratio (SNR) at the peak of its autocorrelation function, and is consequently optimum for detecting the signal in noise.

A very common radar waveform is the Linear FM (LFM) chirp signal. Its utility is that it is fairly readily generated by a variety of technologies, and is easily processed by a variety of techniques that ultimately implement a Matched Filter, or nearly so. However, since a LFM chirp waveform has nearly a rectangular PSD, its autocorrelation function exhibits a sinc() function shape, with its attendant problematic sidelobe structure.

Reducing the sidelobes of the Matched Filter output (actually increasing the peak to sidelobe ratio) is typically accomplished by linear filtering the output, most often by applying window functions or data tapering. This additional filtering perturbs the Matched Filter result to reduce sidelobes as desired. However, since the cumulative filtering is no longer precisely matched to the signal, it necessarily reduces output SNR as well, typically by 1-2 dB (depending on the filtering or weighting function used). ${ }^{1}$

It is well-known that Non-Linear FM (NLFM) chirp modulation can advantageously shape the PSD such that the autocorrelation function exhibits substantially reduced sidelobes from its LFM counterpart. Consequently, no additional filtering is required and maximum SNR performance is preserved. However precision NLFM chirps are more difficult to design, produce, and process.

Alternatives to NLFM modulation for the purpose of shaping the PSD, such as amplitude tapering the transmitted signal, are not viable since typically efficient power

amplification of the waveform necessitates operating the hardware in a nonlinear manner, e.g. operating the amplifiers in compression. This substantially reduces the ability to maintain precision amplitude tapering. Waveform phase remains unaffected by operating amplifiers in compression.

What is desired by a radar designer is then a NLFM waveform that is 1) easily produced, 2) easily processed, and 3) easily designed to meet target performance criteria, including bandwidth constraints and sidelobe reduction goals.

The progress of technology now offers the possibility of addressing the first two points, namely easily producing and processing the NLFM waveform. The advent of high-speed Digital-to-Analog Converters (DACs) and high-speed large-scale Field Programmable Gate Arrays (FPGAs) currently facilitate generating high-performance precision digital LFM chirp waveforms. ${ }^{2,3}$ This suggest that more exotic waveforms might now be within the realm of possibilities. These same FPGAs and high-speed Analog-to-Digital 
Converters (ADCs) allow directly sampling fairly wide bandwidth signals. Modern highspeed processors allow more complex filtering and detection algorithms to be employed.

The literature discusses NLFM waveform design for the purpose of sidelobe mitigation.

Johnston and Fairhead ${ }^{4}$ review the existing literature on NLFM waveforms circa 1986, and then proceed to outline a waveform design technique. They also discuss the Doppler sensitivity of these waveforms.

Keel, et al., ${ }^{5}$ discuss a step frequency waveform employing nonlinear frequency steps. Griffiths and Vinagre ${ }^{6}$ provide one procedure for designing a piecewise linear NLFM chirp waveform. DeWitte and Griffiths ${ }^{7}$ later extend this to continuous NLFM waveforms.

Varshney and Thomas ${ }^{8}$ explore several techniques for sidelobe reduction and conclude "[o]verall, NLFM has better detection rate characteristics and is more accurate in range determination than LFM" as well as the other techniques studied. Cook, et al., ${ }^{9}$ discuss matched filter responses to NLFM waveforms.

Butler ${ }^{10}$ discusses NLFM chirp waveform generation with Surface Acoustic Wave (SAW) dispersive filters.

Collins and Atkins ${ }^{11}$ discuss NLFM waveforms applied to active sonar signals.

We also note that NLFM waveform design and analysis is interestingly very related to the laser beam shaping problem, as presented in Dickey and Holswade. ${ }^{12}$

However, connecting the NLFM radar waveform that is designed to one that is easily produced seems generally overlooked.

\section{Overview \& Summary}

We propose to generate a NLFM waveform by using a cascaded integrator/accumulator structure. Several specific architectures are examined to meet target performance criteria, including bandwidth constraints and sidelobe reduction goals.

We first examine a fixed parameter set to generate a fixed polynomial phase function. Polynomial coefficients are selected to be constant during the pulse.

Alternatively, a NLFM waveform can be generated via integrating a stepped parameter set, whereby parameters are constant over specific intervals, with the pulse width encompassing multiple intervals. The parameter changes in steps during the course of the pulse as a function of time.

Alternatively, the parameter steps can be made a function of the pulse's instantaneous frequency. 


\section{Detailed Analysis}

\subsection{General Principles of NLFM chirps}

To facilitate a comparison, consider first a conventional Linear FM (LFM) chirp with characteristics in figure 1 . Note that the frequency ramp is linear, and the spectrum is flat-topped with steep sides, nearly a rectangle. Note also that the Impulse Response (IPR) is expected to be nearly a sinc() function with $-13 \mathrm{~dB}$ sidelobes.

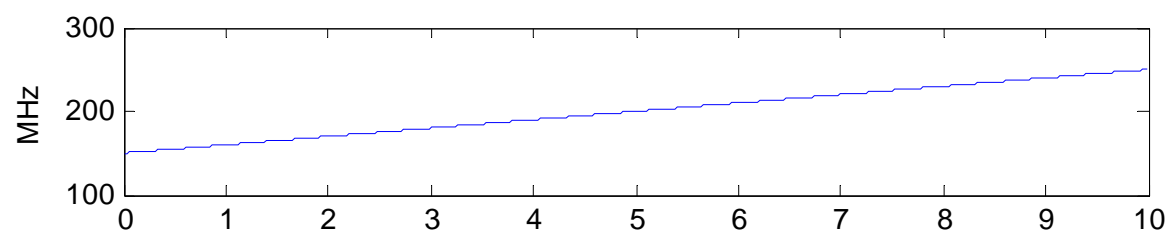

(a) usec
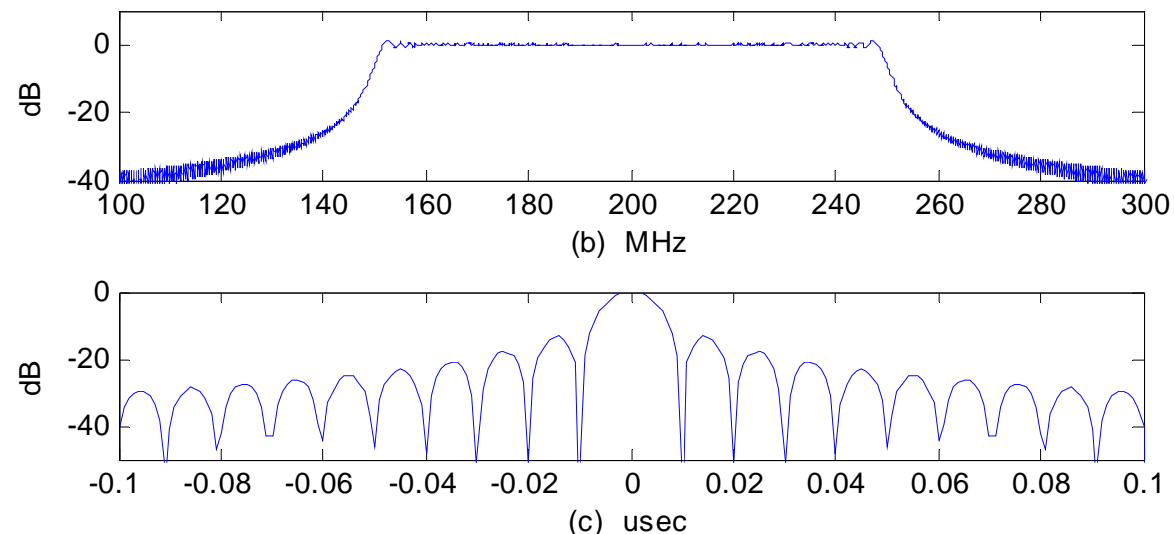

Figure 1. Example LFM chirp with (a) frequency vs. time, (b) magnitude spectrum, and (c) time autocorrelation function.

Now consider the Non-linear FM (NLFM) chirp with characteristics in figure 2. Note here that the frequency ramp is non-linear, with steeper slope at the beginning and at the end of the pulse. The corresponding spectrum is tapered with lower magnitude at its edges. This spectral shaping results in the autocorrelation function exhibiting attenuated sidelobes, limited to less than $-35 \mathrm{~dB}$. Furthermore these characteristics are achieved without any SNR-robbing sidelobe filtering or window functions. 


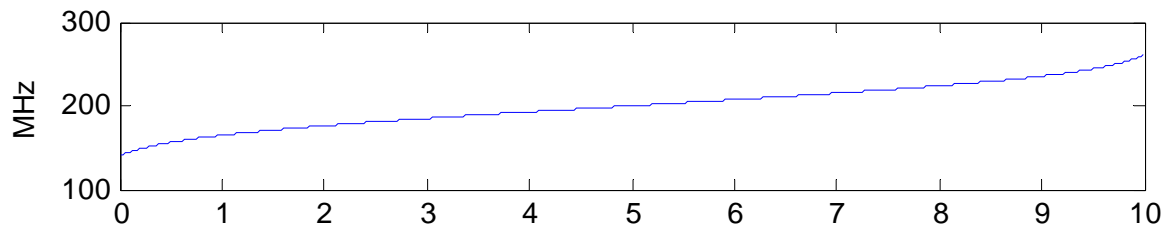

(a) usec

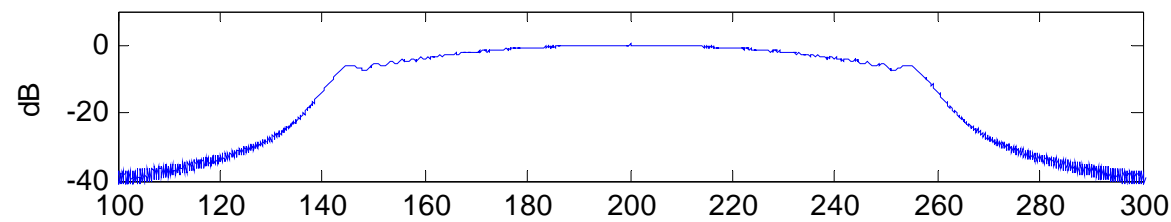

(b) $\mathrm{MHz}$

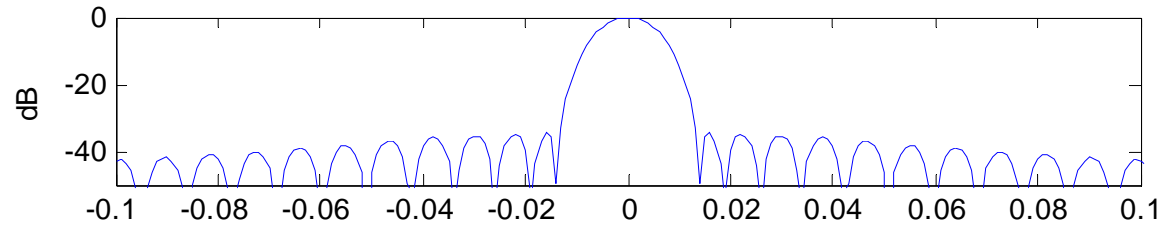

(c) usec

Figure 2. Example NLFM chirp with (a) frequency vs. time, (b) magnitude spectrum, and (c) time autocorrelation function.

\subsubsection{Finding desired chirp rate function of time}

We limit our investigation to signals with large time-bandwidth products, which are typical for high-performance radar systems.

Rayleigh energy criteria infer that for a LFM chirp of a constant bandwidth, that PSD must be proportional to pulse width. Consequently, under conditions of constant bandwidth, the PSD must be inversely proportional to chirp rate.

Furthermore, the principle of stationary phase infers that "the major contribution to the spectrum at any frequency $\omega$ is made by that part of the signal which has instantaneous frequency $\omega$. ." This means that for a NLFM chirp, that the PSD at a particular frequency is inversely proportional to the chirp rate at that particular frequency.

We begin by defining a generic radar waveform, perhaps an FM chirp, as

$$
X(t)=\operatorname{rect}\left(\frac{t}{T}\right) \exp j \Phi(t)
$$

where, 


$$
\begin{aligned}
& t=\text { time, } \\
& T=\text { pulse width, } \\
& \operatorname{rect}(z)=\left\{\begin{array}{cc}
1 & |z| \leq 1 / 2 \\
0 & \text { else }
\end{array} .\right.
\end{aligned}
$$

The instantaneous frequency is related to phase as

$$
\omega(t)=\frac{d}{d t} \Phi(t)
$$

and the instantaneous chirp rate is related to frequency as

$$
\gamma(t)=\frac{d \omega(t)}{d t}
$$

For a generic chirp signal, the phase becomes

$$
\Phi(t)=c_{0}+\omega_{0} t+\iint \gamma(t) d t d t
$$

where

$$
\begin{aligned}
& c_{0}=\text { reference phase, and } \\
& \omega_{0}=\text { reference frequency } .
\end{aligned}
$$

We note that $\gamma(t)$ is the component of $\Phi(t)$ that makes it a chirp. Furthermore, if $\gamma(t)=\gamma_{0}$ for some constant $\gamma_{0}$ then this reduces to the LFM case. For the NLFM case we expect useful $\gamma(t)$ to be predominantly " $U$ " shaped, indicating greater chirp rates at the start and end of a pulse compared to that at the middle. This will in turn cause a tapering of the PSD at the band edges. We also expect that a symmetric PSD will require a symmetric $\gamma(t)$.

Based on the foregoing analysis, we now identify the relationship of chirp rate to instantaneous frequency as $\gamma_{\omega}(\omega)$ and relate it to a specific window or taper function as

$$
\gamma_{\omega}\left(\omega-\omega_{0}\right)=\frac{\gamma_{\omega}(0)}{W\left(\omega-\omega_{0}\right)} \quad \text { for }-\frac{\Omega}{2} \leq\left(\omega-\omega_{0}\right) \leq \frac{\Omega}{2}
$$

where

$$
\begin{aligned}
& \omega_{0}=\text { the chirp center reference frequency, } \\
& W(\omega)=\text { the desired taper function for the PSD, and } \\
& \Omega=\text { the chirp bandwidth of interest. }
\end{aligned}
$$


We reiterate that $\gamma(t)$ is a function of time, whereas $\gamma_{\omega}(\omega)$ is the chirp rate at a particular frequency $\omega$.

We note that under these conditions $W(0)=1$, and we typically expect $W(\omega)$ to be symmetric about its center.

The task now becomes to find a specific $\Phi(t)$ that yields the desired $\gamma_{\omega}\left(\omega-\omega_{0}\right)$. More specifically, the task now becomes to find a specific $\gamma(t)$ that yields the desired $\gamma_{\omega}\left(\omega-\omega_{0}\right)$.

We also identify at this time from symmetry considerations that $\omega(0)=\omega_{0}$. Consequently

$$
\begin{aligned}
& \gamma_{\omega}(0)=\gamma(0), \\
& \gamma_{\omega}\left(\frac{\Omega}{2}\right)=\gamma\left(\frac{T}{2}\right),
\end{aligned}
$$

and more generally

$$
\gamma_{\omega}\left(\omega(t)-\omega_{0}\right)=\gamma(t)
$$

Consequently we need to solve

$$
\gamma(t)=\frac{\gamma(0)}{W\left(\omega(t)-\omega_{0}\right)}
$$

with the constraint

$$
\int_{-T / 2}^{T / 2} \gamma(t) d t=\Omega .
$$

This suggests the following iterative procedure for finding $\gamma(t)$.

1) select an initial $\gamma(t)$ consistent with a LFM chirp, i.e. $\gamma(t)=\Omega / T$.

2) Integrate $\gamma(t)$ to calculate $\omega(t)$.

3) Adjust $\gamma(t)$ and $\omega(t)$ to meet the $\Omega$ constraint.

4) Calculate $W\left(\omega(t)-\omega_{0}\right)$, and then a new $\gamma(t)$.

5) Repeat steps 2-5 until convergence. 
This procedure was successfully used to design the NLFM chirp of figure 2, using a -35 dB Taylor window.

\subsubsection{Bandwidth}

From communications theory, the well-known Carson's rule states that the bandwidth of a FM modulated signal is approximately twice the sum of the maximum frequency deviation from the carrier plus the modulating frequency. ${ }^{13}$ Consequently, since $\gamma(t)$ itself is typically expected to be low-frequency in nature, then the transmitted signal bandwidth of the NLFM chirp is expected to be approximately the chirp bandwidth

$$
\Omega_{T} \approx \Omega=\omega(T / 2)-\omega(-T / 2)=2\left[\omega(T / 2)-\omega_{0}\right] .
$$

As with LFM chirps, we expect this to be most accurate for signals with large timebandwidth products.

It is expected that the bandwidth increase over that of a LFM chirp will be fractional for a comparable autocorrelation width, similar to that of amplitude tapering.

\section{Doppler Tolerance}

Several papers suggest that an issue for NLFM waveforms is their tolerance to Doppler shifts, i.e., maintaining their desirable sidelobe properties when Doppler shifted.

However Johnson and Fairhead state "the choice of window function [i.e. desired PSD taper for NLFM design] appears less important than previously supposed, although the truncated Gaussian window does give slightly better tolerance than the others to Doppler shift.” Morgan ${ }^{14}$ proposes a hybrid approach to deal with this.

We will not explore this aspect any further in this report. At the time of this writing, a separate report is being prepared to address this. 


\subsection{Polynomial-Phase Chirps}

A conventional LFM chirp signal can be described with quadratic phase function

$$
\Phi(t)=c_{0}+c_{1} t+\frac{c_{2}}{2} t^{2}
$$

where,

$$
\begin{aligned}
& c_{0}=\text { reference phase } \\
& c_{1}=\text { reference frequency, } \\
& c_{2}=\text { nominal constant chirp rate. }
\end{aligned}
$$

This signal phase is easily generated parametrically with a double integration. That is

$$
\left[c_{0}+c_{1} t+\frac{c_{2}}{2} t^{2}\right]=c_{0}+\int\left[c_{1}+\int c_{2} d t\right] d t \text {. }
$$

The digital hardware counterpart to an analog integrator is an accumulator. The resulting phase is translated to an amplitude via a trigonometric lookup-table and applied to a DAC. The resulting analog signal is filtered and utilized.

We examine now an extension of this concept to a higher-order polynomial phase function. Specifically we examine a NLFM chirp signal that can be described with phase function

$$
\Phi(t)=\sum_{n=0}^{N} \frac{c_{n}}{n !} t^{n}
$$

When $c_{n}=0$ for $n>2$, this reduces to the LFM chirp. We also note that for the PSD tapering to be symmetrical, in this formulation $c_{n}=0$ for odd $n>2$, that is, for $n=3$, $7, \ldots$

As with the LMF chirp, this signal has the desirable attribute in that it can be generated parametrically with cascaded integrations or accumulations, the number of accumulators being equal to the order $N$ of the polynomial. An architecture for this is shown in Figure 3. 


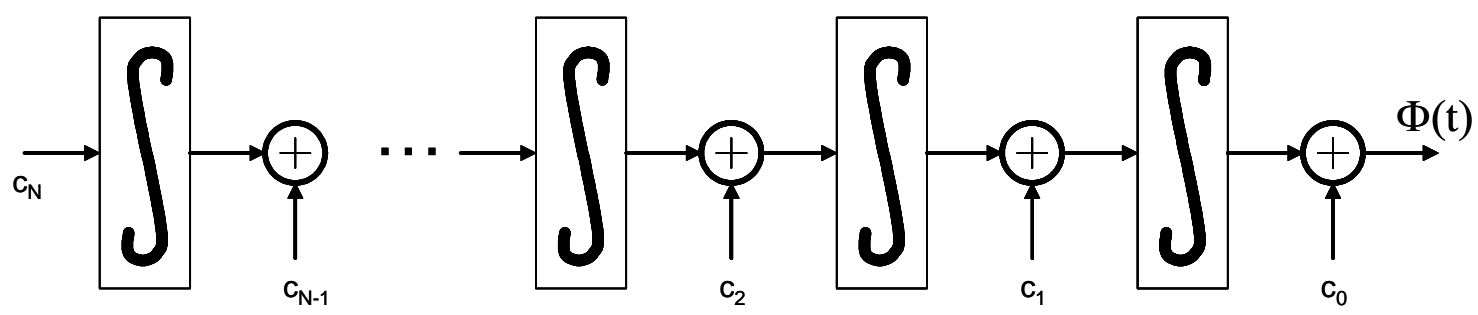

Figure 3. Cascaded integrator architecture for implementing polynomial phase function.

We note that the instantaneous frequency for this signal is

$$
\omega(t)=\frac{d}{d t}\left\{\sum_{n=0}^{N} \frac{c_{n}}{n !} t^{n}\right\}=\sum_{n=1}^{N} \frac{c_{n}}{(n-1) !} t^{n-1} .
$$

Consequently, the bandwidth of the polynomial-phase NLFM chirp is expected to be approximately

$$
\Omega \approx 2\left[\sum_{n=0}^{N} \frac{c_{n}}{(n-1) !}\left(\frac{T}{2}\right)^{n-1}-c_{1}\right] .
$$

The chirp rate is then

$$
\gamma(t)=\frac{d}{d t} \omega(t)=\sum_{n=2}^{N} \frac{c_{n}}{(n-2) !} t^{n-2} .
$$

Clearly, for a phase polynomial of order $N$, we need a chirp rate polynomial of order $(N-2)$.

Some discussion of low-order polynomial phase functions can be found in the paper by Cook, et al. 


\subsubsection{Determining Phase Polynomial Coefficients}

The task is to find phase polynomial coefficients that provide the desired sidelobe reduction.

One seemingly reasonable approach would be to find phase polynomial coefficients that allow acceptable approximation to a known amplitude weighting (window) function. It is desired to accomplish this for a minimal polynomial order $N$. We would expect too high an order $N$ leading to conditioning problems in coefficient calculations. Too low an order $N$ will inadequately model the chirp rate, and hence cause unwanted sidelobe artifacts in the waveform autocorrelation function. Recall that for a LFM chirp $N=2$.

We illustrate results with a number of examples.

Figure 3 shows the chirp rate function and waveform autocorrelation function for a phase polynomial of order 12 fitted to achieve a Taylor weighting with -35 dB sidelobes and $\bar{n}=4$. Figure 4 shows the results of a phase polynomial of order 8 fitted to achieve the same Taylor weighting. Note the elevated sidelobes as the match to the chirp rate becomes less precise.

Other weighting functions can be adequately achieved with lower order polynomial phase functions. Generally weighting functions with higher sidelobes seem to require lower order polynomial phase functions for satisfactory performance. Figure 5 shows good performance with $-30 \mathrm{~dB}$ Taylor weighted sidelobes with a phase polynomial of order 8 . Figure 6 shows good performance with $-20 \mathrm{~dB}$ Taylor weighted sidelobes with a phase polynomial of order 6 . Figures 7 and 8 show good approximation to Gaussian weighted sidelobes with a phase polynomial of order as small as 4 .

We do acknowledge that any implementation of this architecture must contend with problematic aspects of integration and accumulation, including effects of finite precision and accumulation of errors. 


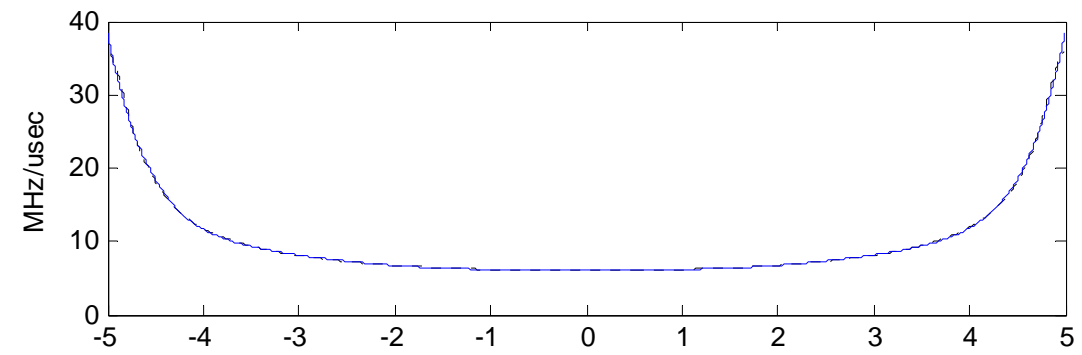

(a) usec

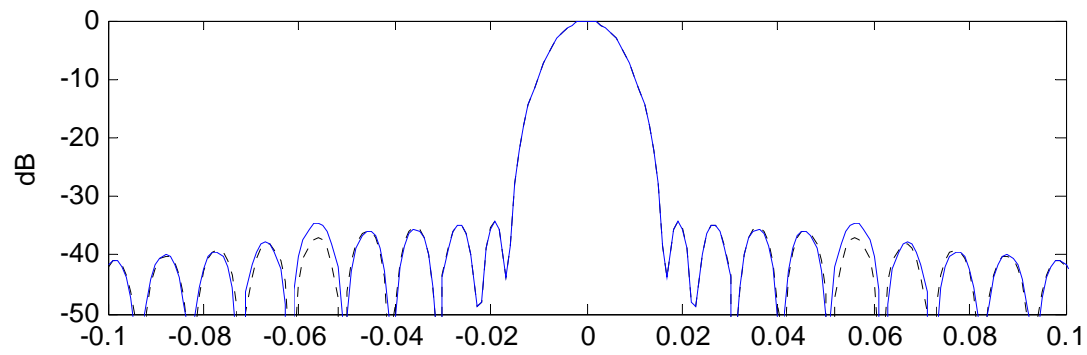

(b) usec

Figure 4. (a) chirp rate function, and (b) autocorrelation function, for NLFM chirp with an order 12 polynomial phase fitted to achieve a Taylor weighting with $-35 \mathrm{~dB}$ sidelobes and $\bar{n}=4$. Dotted lines are ideal, solid lines are actual.

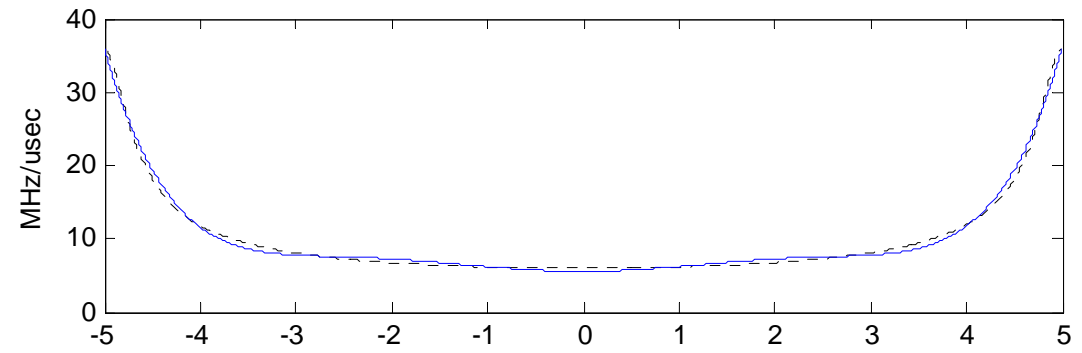

(a) usec

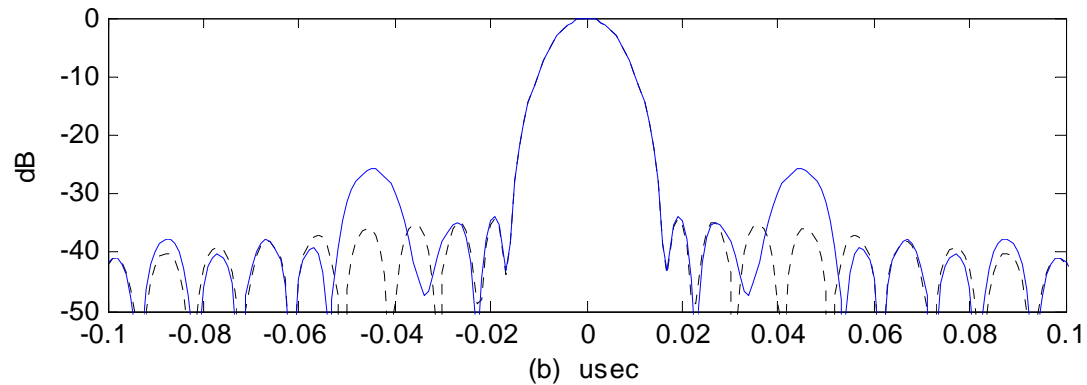

Figure 5. (a) chirp rate function, and (b) autocorrelation function, for NLFM chirp with an order 8 polynomial phase fitted to achieve a Taylor weighting with $-35 \mathrm{~dB}$ sidelobes and $\bar{n}=4$. Dotted lines are ideal, solid lines are actual. 


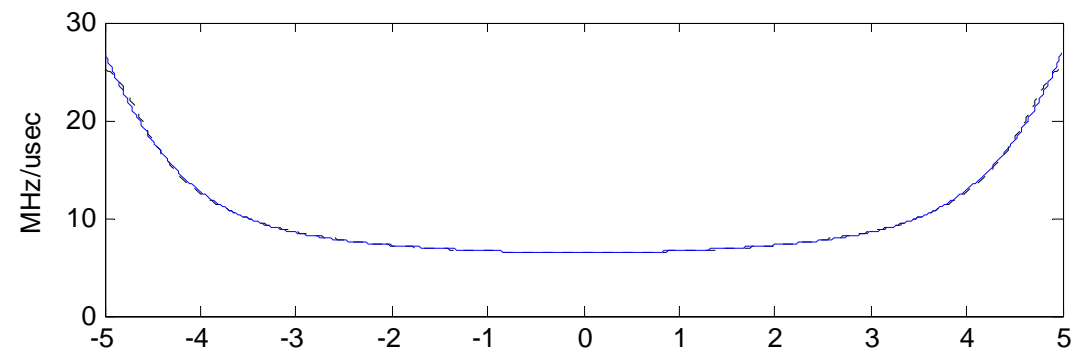

(a) usec

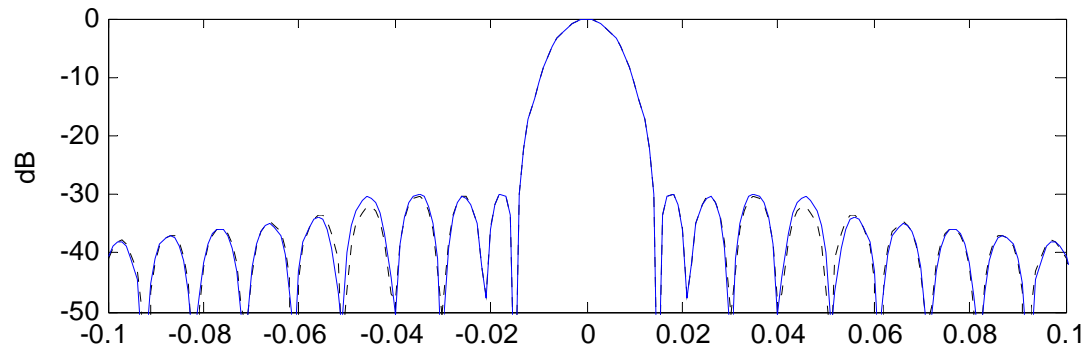

(b) usec

Figure 6. (a) chirp rate function, and (b) autocorrelation function, for NLFM chirp with an order 8 polynomial phase fitted to achieve a Taylor weighting with $-30 \mathrm{~dB}$ sidelobes and $\bar{n}=3$. Dotted lines are ideal, solid lines are actual.

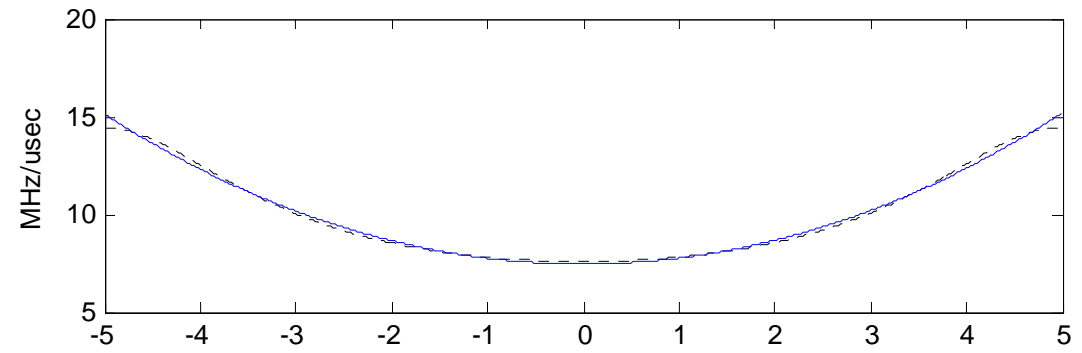

(a) usec

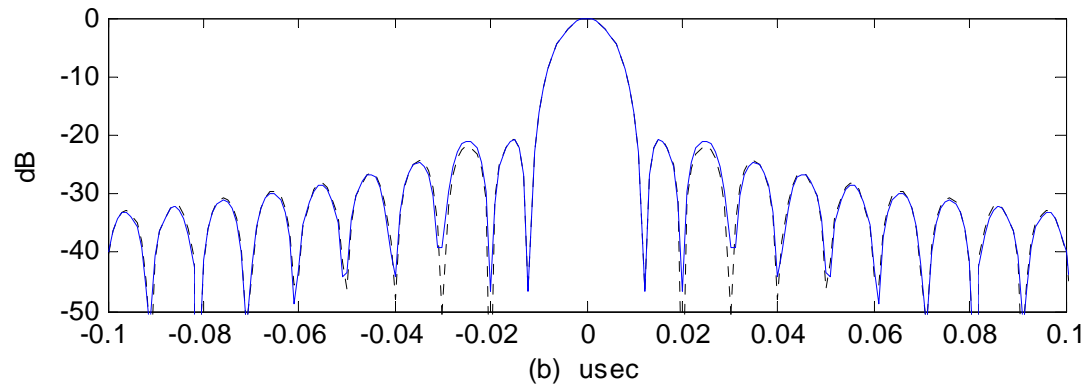

Figure 7. (a) chirp rate function, and (b) autocorrelation function, for NLFM chirp with an order 6 polynomial phase fitted to achieve a Taylor weighting with $-20 \mathrm{~dB}$ sidelobes and $\bar{n}=3$. Dotted lines are ideal, solid lines are actual. 


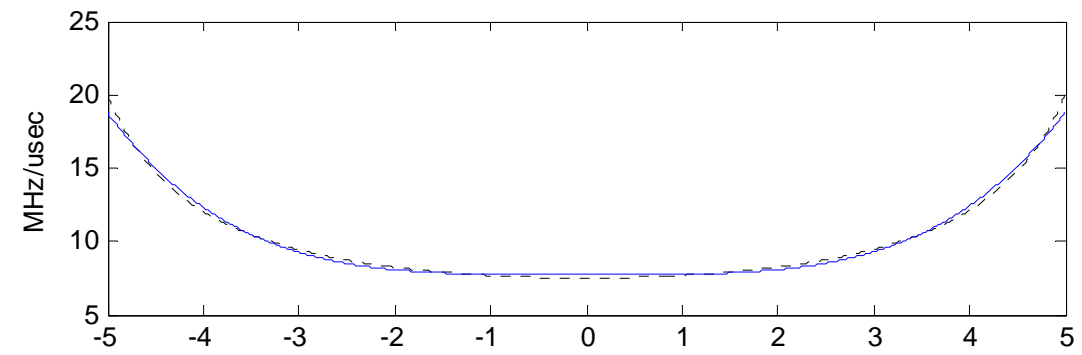

(a) usec

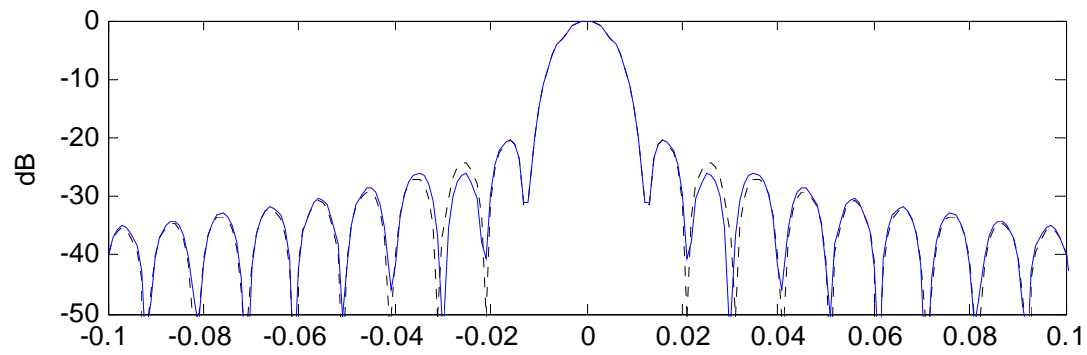

(b) usec

Figure 8. (a) chirp rate function, and (b) autocorrelation function, for NLFM chirp with an order 6 polynomial phase fitted to achieve a Gaussian weighting with $\alpha=1.4$. Dotted lines are ideal, solid lines are actual.

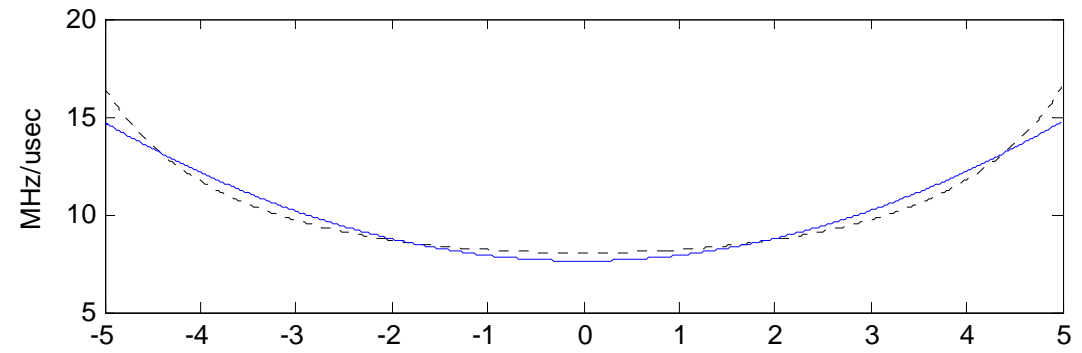

(a) usec

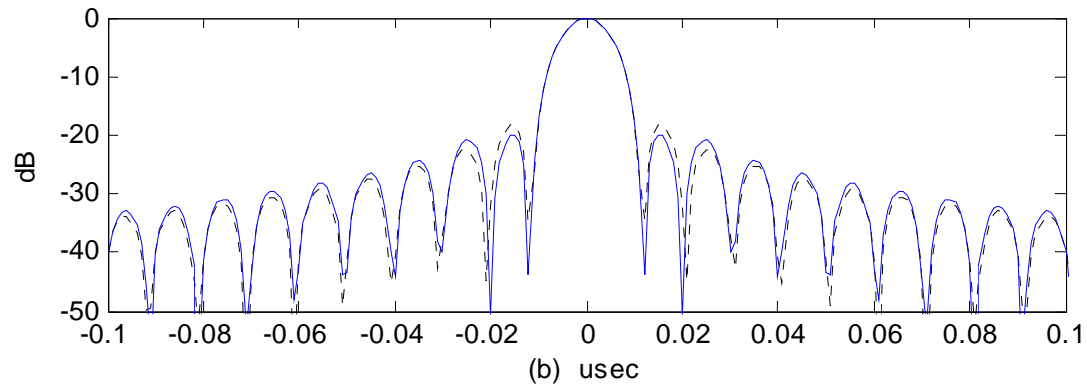

Figure 9. (a) chirp rate function, and (b) autocorrelation function, for NLFM chirp with an order 4 polynomial phase fitted to achieve a Gaussian weighting with $\alpha=1.2$. Dotted lines are ideal, solid lines are actual. 
A question remains, however, "How well can we do with polynomial phase of order $N$ if we don't necessarily try to match a specific weighting function?" This begs the question of whether and how some 'optimum' polynomial can be found for a phase function to generate minimum sidelobe energy in a manner similar to the technique for weighting functions presented by Dickey, et al. ${ }^{15}$ The answer to this question, regardless of how interesting it might be, is however beyond the scope of this report.

\subsection{Stepped-Parameter Chirps}

Consider first a phase function that is described by a polynomial of order $N$. This implies that the $N$ th time derivative of this phase is a constant over the entire pulse width of the waveform. Necessarily, the $(N-1)$ th time derivative is linear.

Now consider the additional degree of freedom of allowing the $N$ th time derivative to be not a single constant, but rather a sequence of constants, each constant being over some finite interval within the pulse width $T$. That is

$$
\frac{d^{N}}{d t^{N}} \Phi(t)=\sum_{m=1}^{M} b_{m} r e c t\left(\frac{t-t_{m}}{\tau_{m}}\right)
$$

where

$$
\begin{aligned}
& m=\text { interval index with } 1 \leq m \leq M \\
& t_{m}=\text { center reference time of the mth interval, } \\
& \tau_{m}=\text { the width of the mth interval, and } \\
& b_{m}=\text { the sequence of constants. }
\end{aligned}
$$

We require the intervals to be non-overlapping and span the pulse width,

$$
\sum_{m=1}^{M} \tau_{m}=T
$$

We note that the $(N-1)$ th time derivative of $\Phi(t)$ is piece-wise linear.

The case $M=1$ degenerates into the polynomial phase function previously discussed. The case where $M$ equals the total number of waveform samples degenerates into an arbitrary phase generator, or more precisely an arbitrary phase-derivative generator. Consequently, of interest are values of $M$ between these extremes. We would expect that this degree of freedom would allow fewer cascaded integrators to be needed to generate a waveform of acceptable fidelity. A more general architecture of cascaded integrators and stepped parameters is illustrated in Figure 10. 


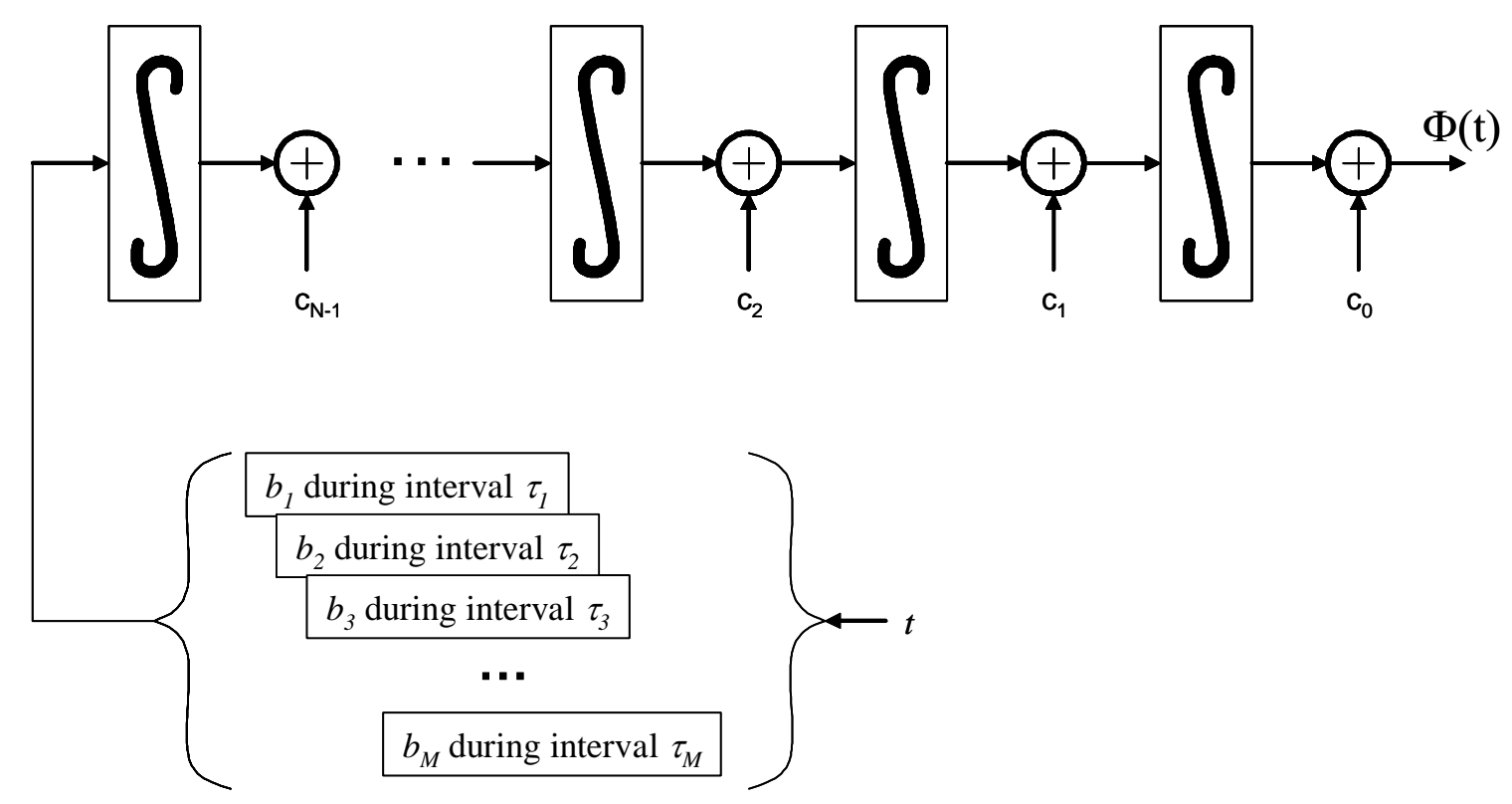

Figure 10. Cascaded integrator architecture for generating stepped parameter chirp waveforms.

Values for $b_{m}$ would be chosen as some function of the desired values of the $N$ th derivative of $\Phi(t)$ over their respective intervals. A representative sample might be used, or perhaps a mean value of all samples within the interval.

Constant interval widths $\tau_{m}$ are also expected to offer some convenience for implementation.

The case for $N=1$ yields the stepped-frequency waveform discussed by Keel, et al.

The case for $N=2$ yields the stepped-chirp (piece-wise linear frequency) waveform discussed by Griffiths and Vinagre.

Consider the following examples, all attempting to generate a $-35 \mathrm{~dB}$ Taylor weighted $(\bar{n}=4)$ autocorrelation function with stepped parameters over equal-width time intervals. Figure 11 shows the case for $N=2$ and $M=10$. Note that the mainlobe is adequately modeled, but sidelobe performance is not adequate. Figure 12 shows the same Taylor weighting and $N=2$, but with $M=40$. Mainlobe and near-in sidelobe performance is good, but distant sidelobe performance is less good. This seems to be a result of how well the chirp rate is matched at the ends of the pulse.

Figure 13 moves the stepped parameter one derivative farther from the phase, namely at $N=3$ with $M=40$, such that the chirp rate is now piece-wise linear. Note that there is now very good match between mainlobes and both near and far sidelobes.

Fewer steps are required for other window functions. For example Figure 14 shows the case for $N=3$ and $M=10$, for generating a $-20 \mathrm{~dB}$ Taylor weighted $(\bar{n}=3)$ autocorrelation function. Note the good match with the mainlobe and all sidelobes. 


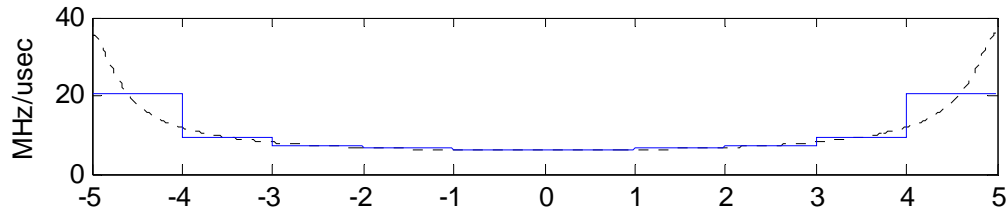

(a) usec

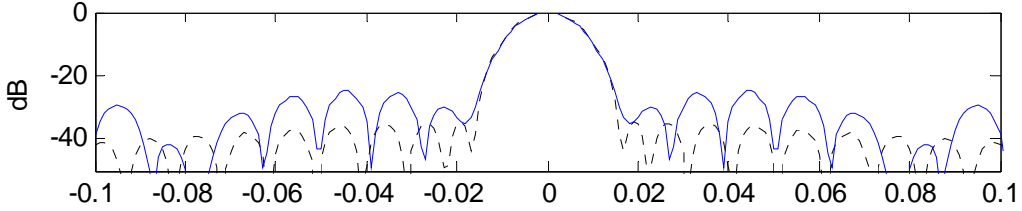

(b) usec

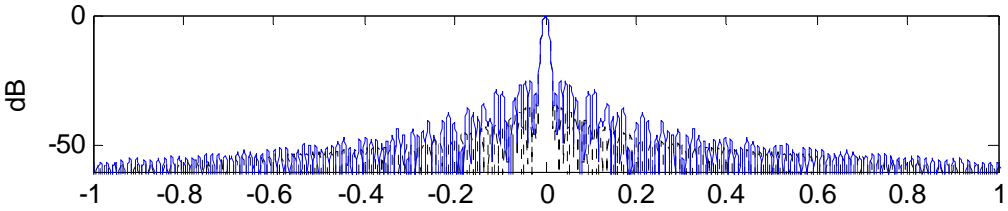

(c) usec

Figure 11. (a) chirp rate function, (b) zoomed autocorrelation function, and (c) expanded autocorrelation function for NLFM chirp with $\mathrm{N}=2$ and $M=10$ to achieve a Taylor weighting with $-35 \mathrm{~dB}$ sidelobes and $\bar{n}=4$. Dotted lines are ideal, solid lines are actual.

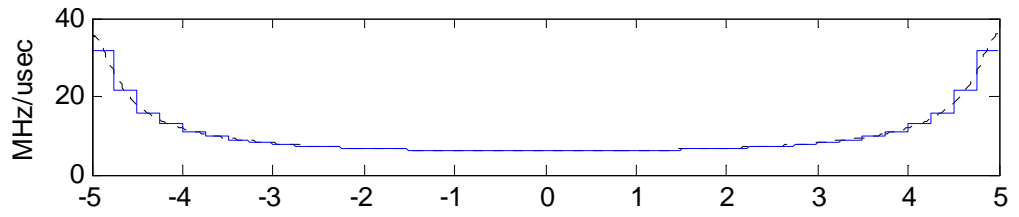

(a) usec

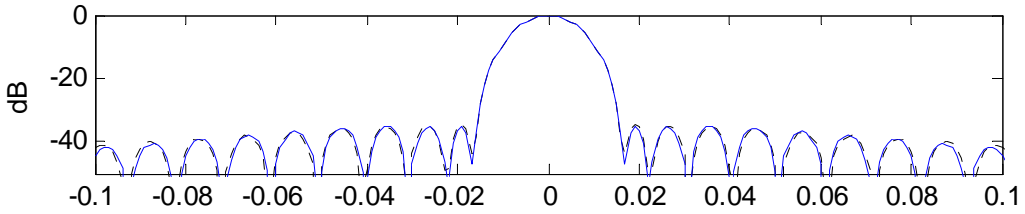

(b) usec

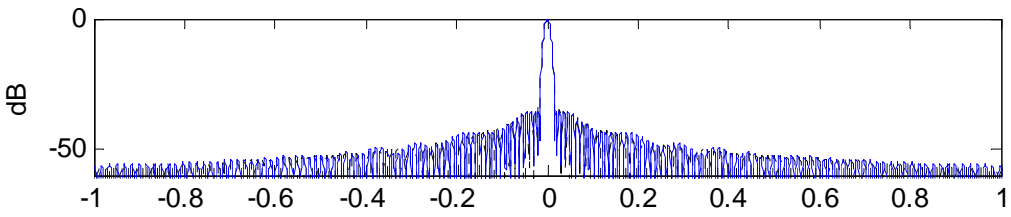

(c) usec

Figure 12. (a) chirp rate function, (b) zoomed autocorrelation function, and (c) expanded autocorrelation function for NLFM chirp with $\mathrm{N}=2$ and $M=40$ to achieve a Taylor weighting with -35 dB sidelobes and $\bar{n}=4$. Dotted lines are ideal, solid lines are actual. 


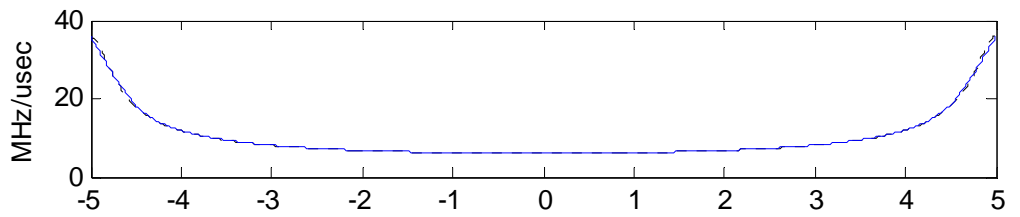

(a) usec

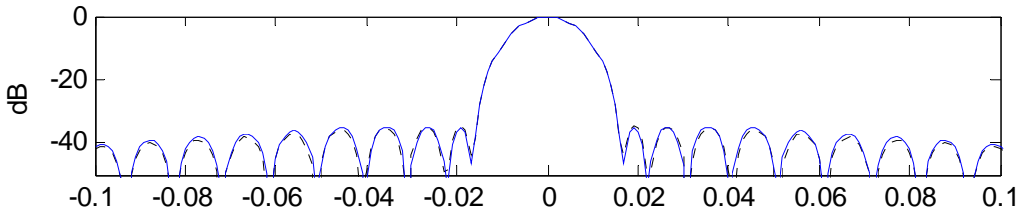

(b) usec

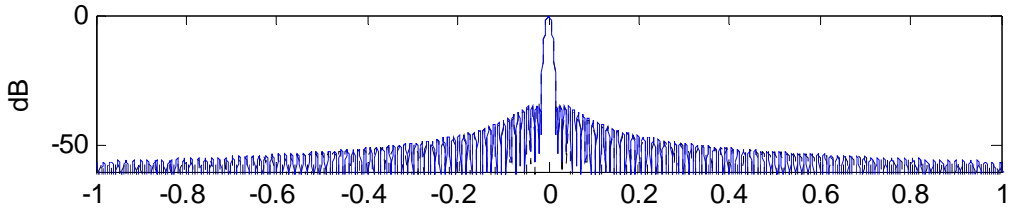

(c) usec

Figure 13. (a) chirp rate function, (b) zoomed autocorrelation function, and (c) expanded autocorrelation function for NLFM chirp with $\mathrm{N}=3$ and $M=40$ to achieve a Taylor weighting with $-35 \mathrm{~dB}$ sidelobes and $\bar{n}=4$. Dotted lines are ideal, solid lines are actual.

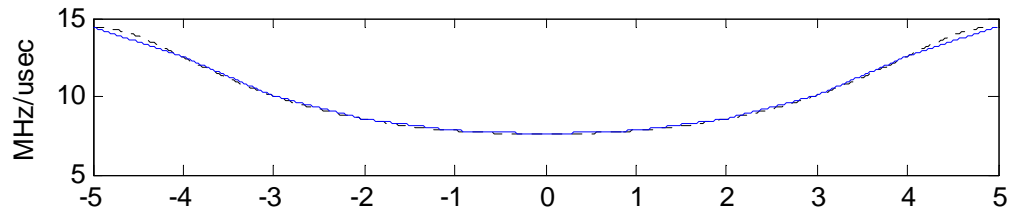

(a) usec

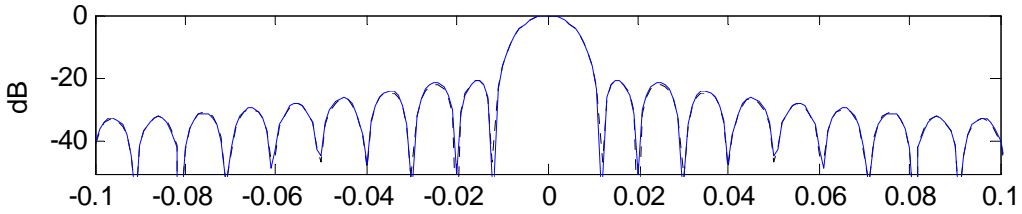

(b) usec

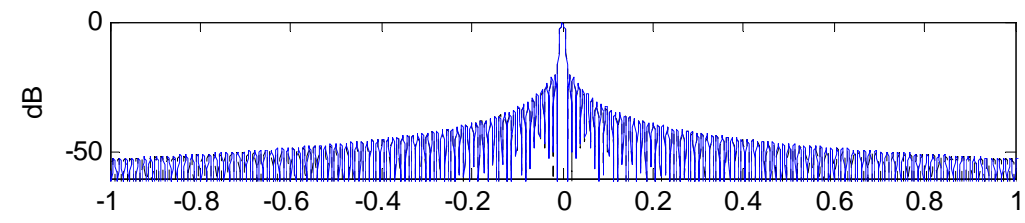

(c) usec

Figure 14. (a) chirp rate function, (b) zoomed autocorrelation function, and (c) expanded autocorrelation function for NLFM chirp with $\mathrm{N}=3$ and $\mathrm{M}=10$ to achieve a Taylor weighting with $-20 \mathrm{~dB}$ sidelobes and $\bar{n}=3$. Dotted lines are ideal, solid lines are actual. 


\subsection{Stepped-Parameter Chirps with Frequency Feedback}

In the previous section we presented analysis of stepped-parameter chirps. We now extend this to the case where $t_{m}$ and $\tau_{m}$ are chosen as a function of instantaneous frequency $\omega(t)$. The driving concept is to adjust parameters more often when parameters are changing more and/or quicker. Since frequency changes faster at beginning and end of the pulse, this seems to be a useful indicator. Consequently, the stepped parameter model becomes

$$
\frac{d^{N}}{d t^{N}} \Phi(t)=\sum_{m=1}^{M} b_{m} \operatorname{rect}\left(\frac{\omega(t)-\omega_{m}}{\Omega_{m}}\right),
$$

where

$$
\begin{aligned}
& m=\text { interval index with } 1 \leq m \leq M \\
& \omega_{m}=\text { center reference frequency of the mth interval, } \\
& \Omega_{m}=\text { the bandwidth of the mth interval, and } \\
& b_{m}=\text { the sequence of constants. }
\end{aligned}
$$

We require the intervals to be non-overlapping in frequency but span the bandwidth,

$$
\sum_{m=1}^{M} \Omega_{m}=\Omega
$$

This architecture is illustrated in figure 15.

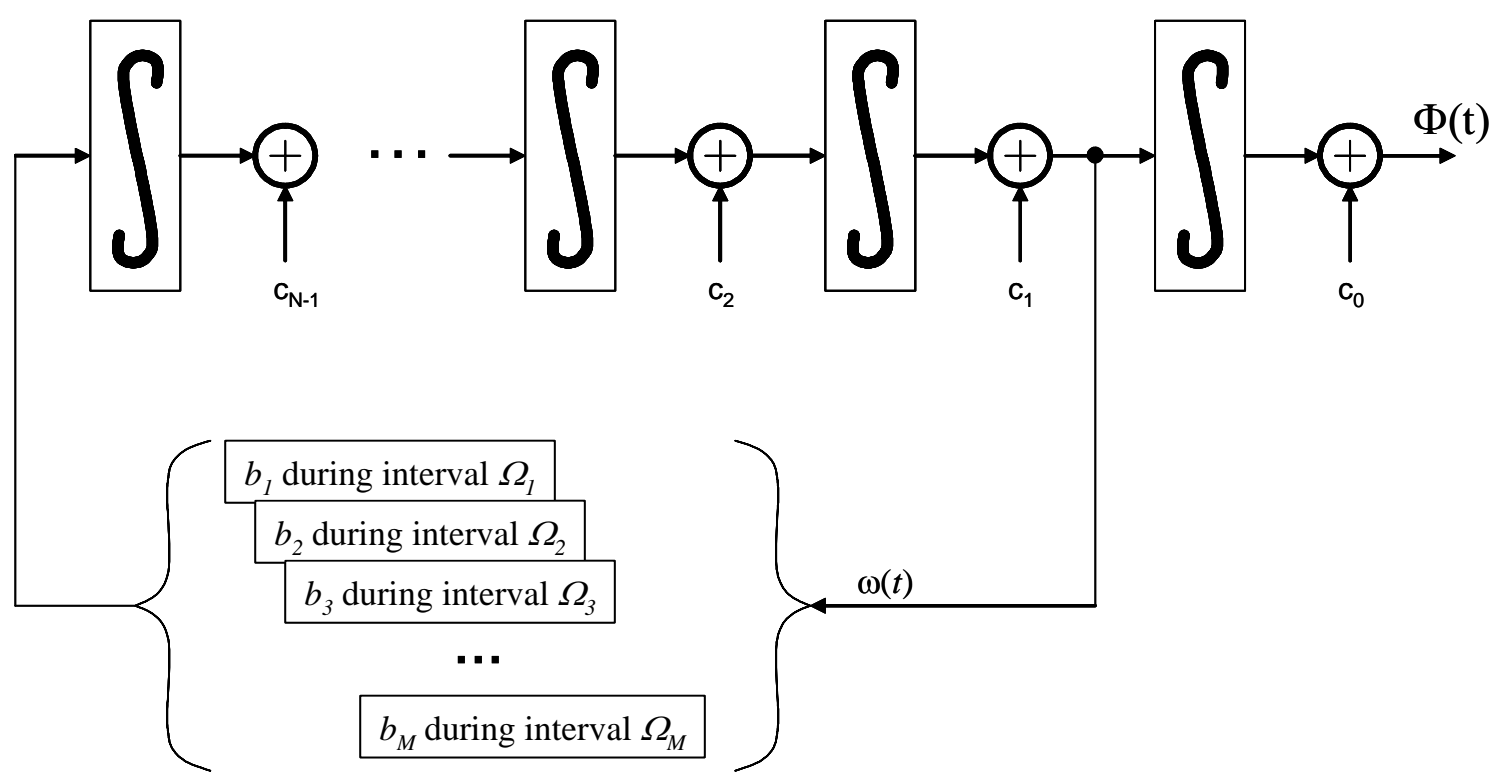

Figure 15. Cascaded integrator architecture for generating stepped parameter chirp waveforms using frequency feedback. 
We note that to be meaningful we require $N \geq 2$.

As before, values for $b_{m}$ would be chosen as some function of the desired values for the $N$ th derivative of $\Phi(t)$ but now over their respective frequency intervals. Some representative sample might be used, or perhaps a mean value of all samples within the interval.

Constant interval widths $\Omega_{m}$ are also expected to offer some convenience for implementation.

Consider the following examples, all attempting to generate a $-35 \mathrm{~dB}$ Taylor weighted $(\bar{n}=4)$ autocorrelation function with stepped parameters over equal-width frequency intervals. Figure 16 shows the case for $N=2$ and $M=10$. Note that the mainlobe is adequately modeled, and near-in sidelobe performance is good, but distant sidelobe performance is problematic. The raised distant sidelobes are an artifact of employing equal frequency intervals, thereby imparting a periodic structure to the PSD. This in turn manifests itself as elevated specific time sidelobes in the autocorrelation function. Figure 17 shows the same Taylor weighting and $N=2$, but with $M=40$. Mainlobe and nearin sidelobe performance is still good, and distant sidelobe performance is substantially improved, although some degradation is still apparent.

Figure 18 moves the stepped parameter one derivative farther from the phase, namely at $N=3$ with $M=40$, such that the chirp rate is now piece-wise linear. Note that there is now very good match between mainlobes and both near and far sidelobes.

As with constant time intervals, fewer frequency steps are required for other window functions. For example Figure 19 shows the case for $N=3$ and $M=10$, for generating a $-20 \mathrm{~dB}$ Taylor weighted $(\bar{n}=3)$ autocorrelation function. Note the good match with the mainlobe and all sidelobes. 


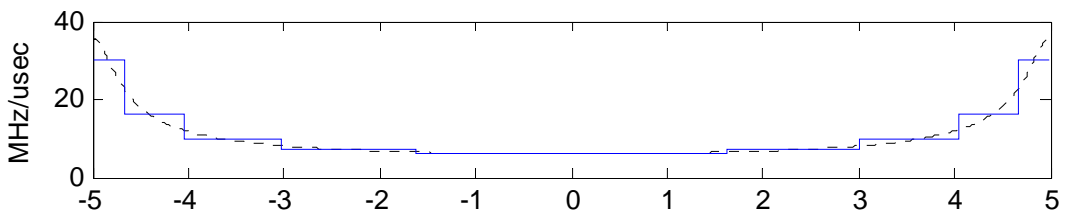

(a) usec

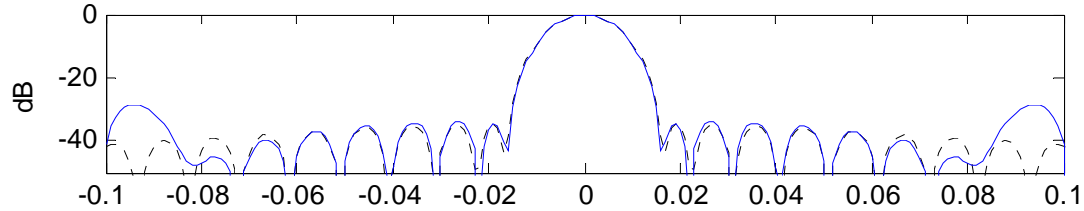

(b) usec

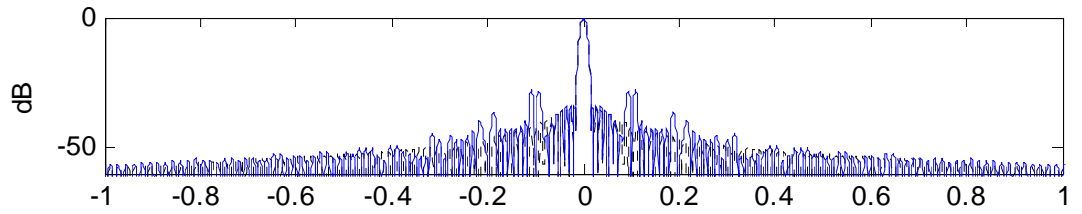

(c) usec

Figure 16. (a) chirp rate function, (b) zoomed autocorrelation function, and (c) expanded autocorrelation function for NLFM chirp with $\mathrm{N}=2$ and $M=10$ to achieve a Taylor weighting with $-35 \mathrm{~dB}$ sidelobes and $\bar{n}=4$. Dotted lines are ideal, solid lines are actual.

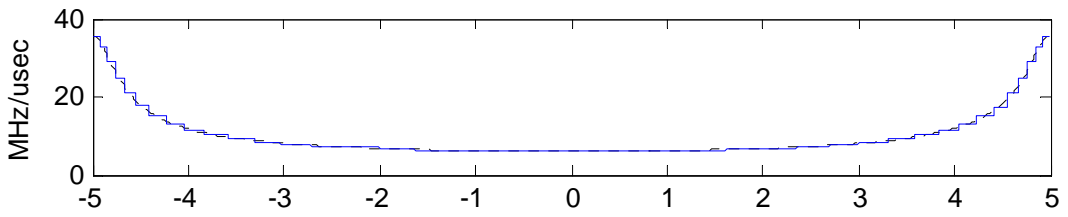

(a) usec

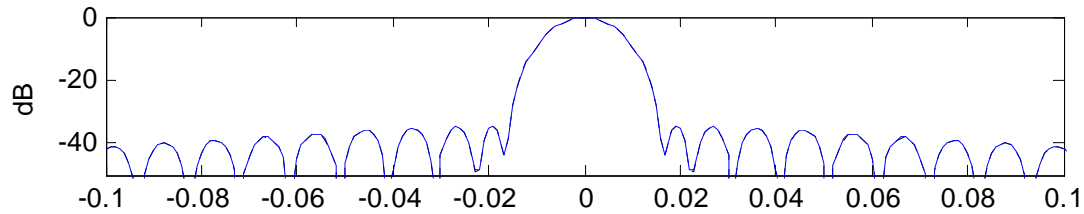

(b) usec

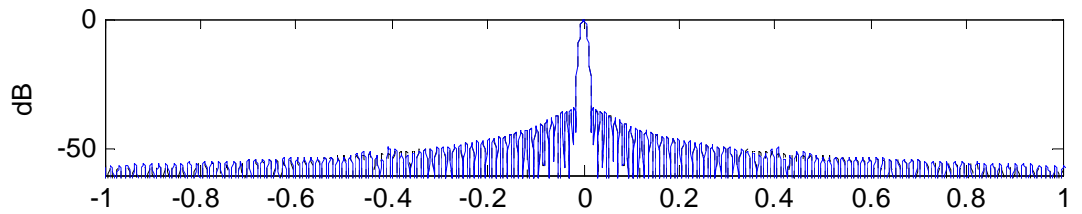

(c) usec

Figure 17. (a) chirp rate function, (b) zoomed autocorrelation function, and (c) expanded autocorrelation function for NLFM chirp with $\mathrm{N}=\mathbf{2}$ and $\mathrm{M}=\mathbf{4 0}$ to achieve a Taylor weighting with -35 dB sidelobes and $\bar{n}=4$. Dotted lines are ideal, solid lines are actual. 


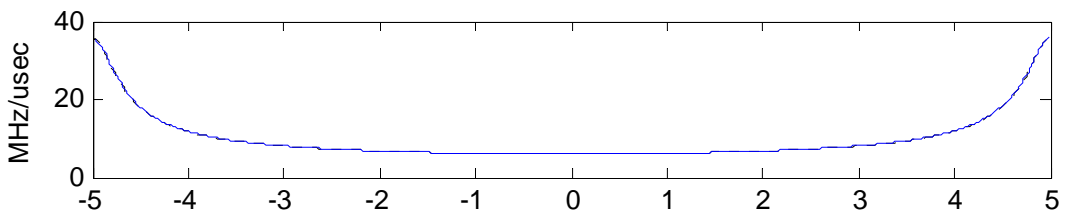

(a) usec

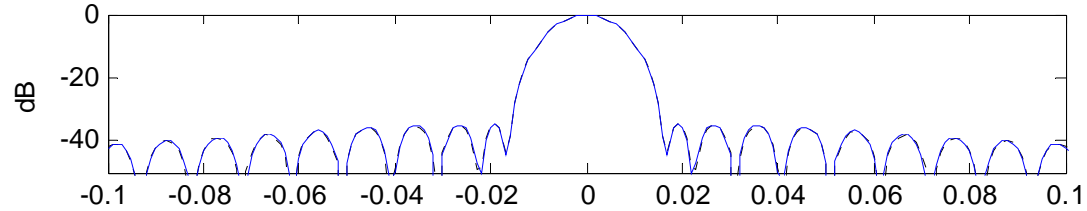

(b) usec

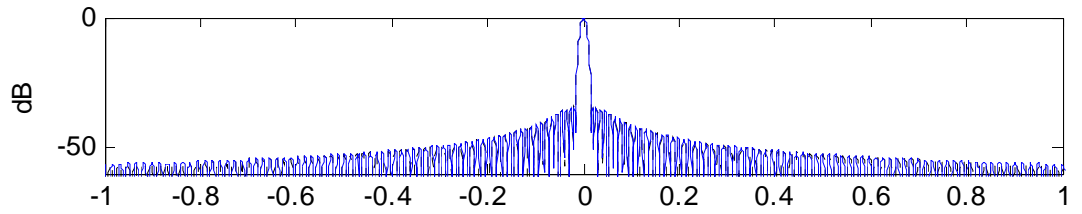

(c) usec

Figure 18. (a) chirp rate function, (b) zoomed autocorrelation function, and (c) expanded autocorrelation function for NLFM chirp with $\mathrm{N}=3$ and $M=40$ to achieve a Taylor weighting with $-35 \mathrm{~dB}$ sidelobes and $\bar{n}=4$. Dotted lines are ideal, solid lines are actual.

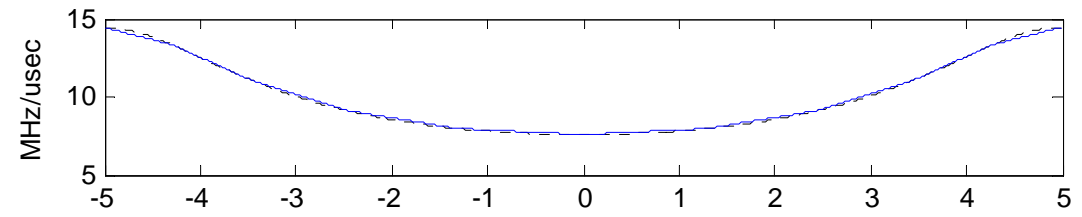

(a) usec

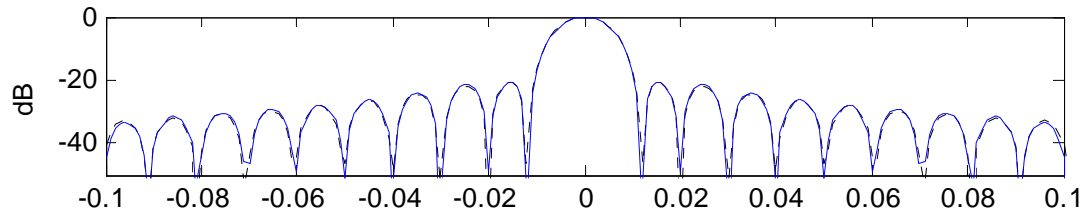

(b) usec

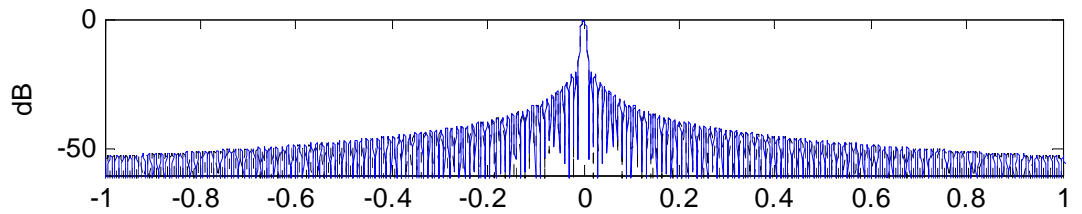

(c) usec

Figure 19. (a) chirp rate function, (b) zoomed autocorrelation function, and (c) expanded autocorrelation function for NLFM chirp with $\mathrm{N}=3$ and $\mathrm{M}=10$ to achieve a Taylor weighting with $-20 \mathrm{~dB}$ sidelobes and $\bar{n}=3$. Dotted lines are ideal, solid lines are actual. 


\subsection{Other Architectures}

In the most general sense, whereas a LFM waveform needs a constant but non-zero chirp rate, a NLFM waveform needs a non-constant chirp rate. Consequently, some mechanism for adjusting chirp rate as a function of time is required. Since instantaneous frequency is also a function of time, and typically in a monotonic fashion, the chirp rate could be effectively adjusted as some function of instantaneous frequency either instead of, or in addition to time. These observations are captured in the general phase-function generating architecture illustrated in figure 20.

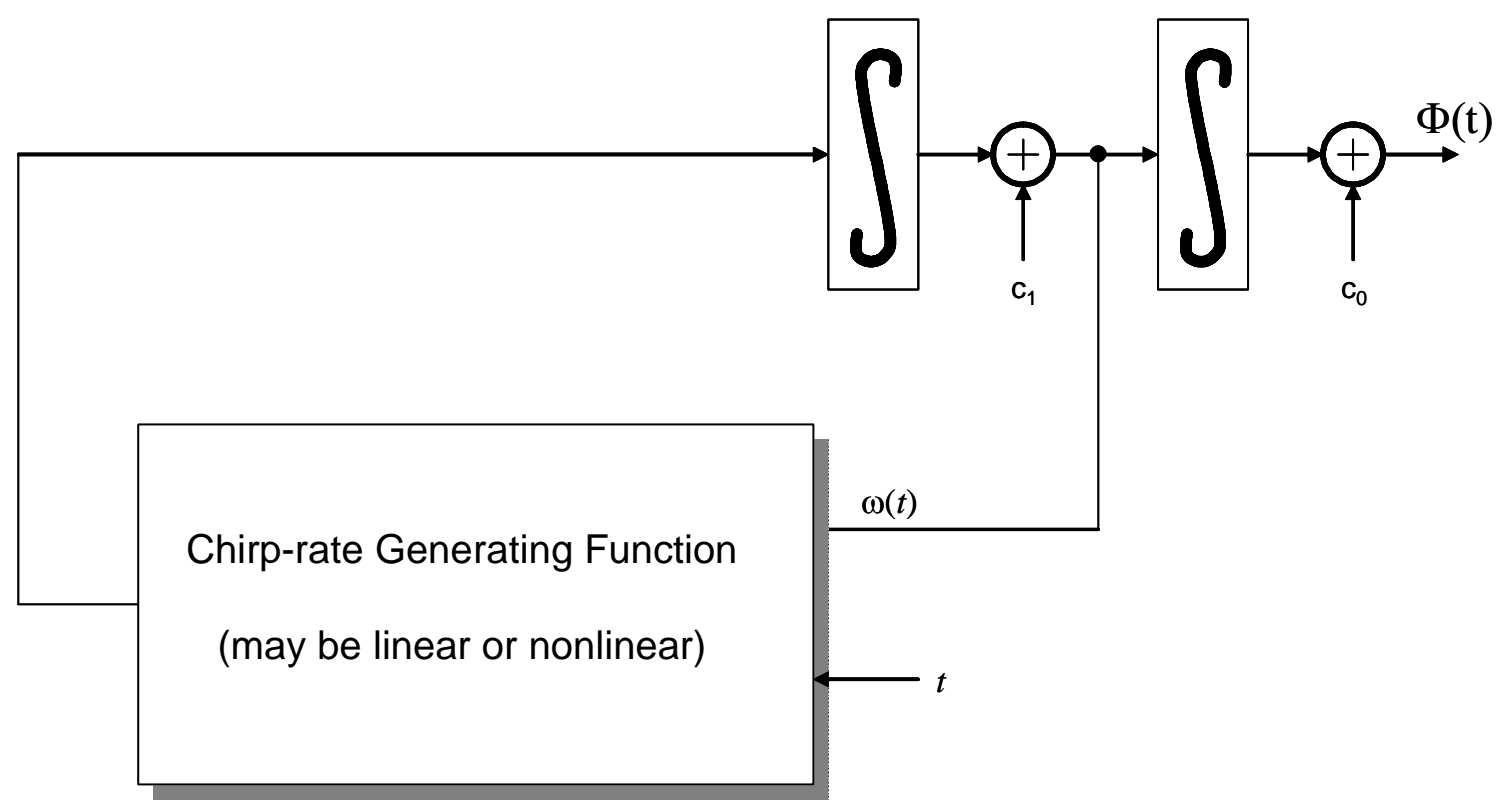

Figure 20. Generalized architecture for NLFM phase generating function. The chirp rate is some function of time and/or instantaneous frequency.

The chirp-rate generating function may be either linear or nonlinear, continuous or discontinuous, with derivatives that may exist or not. Earlier examples in this report showed a polynomial function, parameters that stepped with time, and parameters that stepped with instantaneous frequency. Indeed, Collins and Atkins discuss generating an instantaneous frequency with $\tan ($ ) or $\sinh ()$ functions, although no architecture was illustrated or addressed for accomplishing this.

In any case, the simplest technique for creating arbitrary functions in hardware is to use lookup tables. Accumulators functioning as integrators are also rather simple to implement. As the sophistication of hardware resources such as Field Programmable Gate Arrays (FPGAs) increases, then other more exotic functional calculation blocks become available to a designer, offering more options for practical chirp-rate generating functions. 


\section{Conclusions}

The following principal conclusions should be drawn from this report.

- Nonlinear-FM (NLFM) waveforms offer substantial advantages over their LinearFM (LFM) counterparts.

- Generally any practical range sidelobe filtering that can be accomplished with window functions, can also be accomplished by selecting a corresponding NLFM waveform. Matched filter output results will be indistinguishable, except for an increase in SNR using the NLFM waveform.

- The design procedure for a NLFM waveform is straight-forward and presented herein.

- Hardware architectures for generating suitable NLFM waveforms are also straight-forward, with several options presented herein.

- A number of simulation examples are provided herein to illustrate and validate these concepts. 
Matlab files used:

nonlinchirp.m 


\section{REFERENCES}

1 A. W. Doerry, "Performance Limits for Synthetic Aperture Radar - second edition”, Sandia Report SAND2006-0821, Unlimited Release, February 2006.

2 P. A. Dudley, B. L. Tise, “Multiplexed chirp waveform synthesizer”, U.S. Patent 6,614,813, September 2, 2003.

3 D. F. Dubbert, “A High Speed Quadrature Error Corrected Chirp Synthesizer Module for SAR Application”, Workshop on Synthetic Aperture Radar Technology”, Redstone Arsenal, AL, October 16 \& 17, 2002.

${ }^{4}$ J. A. Johnston, A. C. Fairhead, "Waveform design and Doppler sensitivity analysis for nonlinear FM chirp pulses”, IEE Proceedings F (Communications, Radar and Signal Processing), Vol. 133, No. 2, p. 163-175, April 1986.

${ }^{5}$ Byron M. Keel, Jay A. Saffold, Mark R. Walbridge, John Chadwick, "Non-linear stepped chirp waveforms with sub-pulse processing for range sidelobe suppression", Proceedings of the SPIE - The International Society for Optical Engineering Conference on Radar Sensor Technology III, Orlando, Florida, USA, Vol. 3395, p. 87-98, 16 April 1998.

${ }^{6}$ H. D. Griffiths, L. Vinagre, "Design of low-sidelobe pulse compression waveforms", Electron. Lett., vol. 30, no. 12, p. 1004-1005, 1994.

${ }^{7}$ E. De Witte, H. D. Griffiths, "Improved ultra-low range sidelobe pulse compression waveform design”, Electronics Letters, vol. 40, no. 22, p. 1448-50, 28 Oct. 2004.

${ }^{8}$ Lav R. Varshney, Daniel Thomas, “Sidelobe Reduction for Matched Filter Range Processing”, Proceedings of the 2003 IEEE Radar Conference, Huntsville, AL, USA, p. 446-451, 5-8 May 2003.

${ }^{9}$ C. E. Cook, M. Bernfeld, C. A. Palmieri, "Matched filtering, pulse compression and waveform design - IV”, Microwave Journal, vol.8, no.1, p. 73-88, Jan. 1965.

${ }^{10}$ M. B. N. Butler, "Radar applications of s.a.w. dispersive filters”, IEE Proceedings F (Communications, Radar and Signal Processing) vol.127, no.2, p. 118-24, April 1980.

${ }^{11}$ T. Collins, P. Atkins, "Nonlinear frequency modulation chirps for active sonar”, IEE Proceedings on Radar, Sonar, and Navigation, Vol. 146, No. 6, p. 312-316, December 1999. 
${ }^{12}$ F. M. Dickey, Scott C. Holswade, Laser Beam Shaping Theory and Techniques, ISBN 0-8247-0398-7, Marcel Dekker, Inc., 2000.

${ }^{13}$ R. E. Ziemer, W. H. Tranter, Principles of Communications - Systems, Modulation, and Noise, Fourth Edition, ISBN 0-471-12496-6, John Wiley \& Sons, Inc., 1995.

${ }^{14}$ D. P. Morgan, "Nonlinear chirp radar waveforms with improved tolerance to Doppler shifts”, IEE Colloquium on 'Physics and Device Applications of Acoustic Waves' (Digest No.69), p. 8/1-5, London, UK, 22 May 1987.

${ }^{15}$ Fred M. Dickey, Louis A. Romero, Armin W. Doerry, "SAR Window Functions: A Review and Analysis of the Notched Spectrum Problem', Sandia Report SAND20022949, September 2002. 
“To invent, you need a good imagination and a pile of junk."

Thomas A. Edison (1847 - 1931) 


\section{DISTRIBUTION}

Unlimited Release

$1 \quad$ MS 1330

B. L. Remund $\quad 5340$

$1 \quad$ MS 1330

B. L. Burns 5340

$1 \quad$ MS 0503

T. J. Mirabal 5341

1 MS 1330

J. G. Baldwin $\quad 5341$

1 MS 1330

J. C. Lyle

5341

1 MS $1330 \quad$ W. H. Hensley 5342

1 MS $1330 \quad$ T. P. Bielek 5342

1 MS $1330 \quad$ A. W. Doerry 5342

1 MS $1330 \quad$ D. W. Harmony 5342

1 MS $1330 \quad$ J. A. Hollowell 5342

1 MS $1330 \quad$ S. S. Kawka 5342

1 MS $1330 \quad$ M. S. Murray 5342

1 MS $0519 \quad$ B. G. Rush 5342

1 MS 1330 D. G. Thompson 5342

1 MS $1330 \quad$ K. W. Sorensen 5345

1 MS $1330 \quad$ J. A. Bach 5345

1 MS $1330 \quad$ D. F. Dubbert 5345

1 MS $1330 \quad$ G. R. Sloan 5345

1 MS $0519 \quad$ L. M. Wells 5354

1 MS $0519 \quad$ D. L. Bickel 5354

1 MS $0519 \quad$ J. T. Cordaro 5354

1 MS $0518 \quad$ J. M. DeLaurentis 5354

1 MS $1330 \quad$ S. M. Becker 5348

1 MS $1330 \quad$ P. A. Dudley 5348

1 MS $1330 \quad$ P. G. Ortiz 5348

1 MS $1330 \quad$ D. C. Sprauer 5348

1 MS $1330 \quad$ B. L. Tise 5348

2 MS $9018 \quad$ Central Technical Files 8944

2 MS 0899 Technical Library 4536 